\title{
A Sugar Gustatory Receptor Identified from the Foregut of Cotton Bollworm Helicoverpa armigera
}

\author{
Wei Xu • Hui-Jie Zhang • Alisha Anderson
}

Received: 12 September 2012 /Revised: 5 November 2012 / Accepted: 6 November 2012 /Published online: 6 December 2012

(C) The Author(s) 2012. This article is published with open access at Springerlink.com

\begin{abstract}
Helicoverpa armigera (Hübner) is one of the most polyphagous and cosmopolitan pest species, the larvae of which feed on numerous important crops. The gustatory system is critical in guiding insect feeding behavior. Here, we identified a gustatory receptor from $H$. armigera, HaGR9, which shows high levels of identity to DmGR43a from Drosophila melanogaster and BmGR9 from Bombyx mori. Reverse transcriptase PCR (RT-PCR) revealed HaGR9 is highly expressed in larval foregut, with little or no expression in other chemosensory tissues. Membrane topology studies indicated that, like two previously studied B. mori GRs, BmGR8 and BmGR53, HaGR9 has an inverted topology relative to G protein-coupled receptors (GPCRs), an intracellular N-terminus and an extracellular $\mathrm{C}$-terminus. Calcium imaging studies confirmed HaGR9 is a sugar receptor showing dose-dependent responses to D-galactose, D-maltose, and $\mathrm{D}$-fructose. This highly-expressed foregut-specific gustatory receptor may contribute to the regulation of larval feeding behavior.
\end{abstract}

Keywords Helicoverpa armigera · Gustatory receptor . Foregut · Sugar receptor

\section{Introduction}

Insects display strong feeding preferences. The gustatory system plays a critical role in guiding insect feeding behavior. Gustatory stimuli from the environment are recognized

W. Xu $\cdot$ H.-J. Zhang $\cdot$ A. Anderson $(\bowtie)$

CSIRO Ecosystem Sciences, Black Mountain, Australian Capital

Territory 2601, Australia

e-mail: Alisha.Anderson@csiro.au by gustatory receptors (GRs), which are located in the gustatory sensilla distributed throughout the insect body. Despite a growing body of knowledge about the insect gustatory system, little is known about the molecular and cellular mechanisms that underlie recognition of gustatory signals.

Insect GR genes were first identified from Drosophila melanogaster genome based on a bioinformatics approach (Clyne et al., 2000). These proteins were found by using algorithms to search for seven-transmembrane domains, but they are strikingly different and share no sequence similarity with vertebrate GRs (Clyne et al., 2000). Furthermore, the topology is inverted compared to the classic G-Protein Coupled Receptors (GPCRs) (Benton et al., 2006; Robertson and Wanner, 2006; Zhang et al., 2011). Insect gustatory receptors have been classified into "GR43a-like" (Sato et al., 2011), " $\mathrm{CO}_{2}$ " (Jones et al., 2007), "sugar" ( Dahanukar et al., 2001, 2007; Chyb et al., 2003; Jiao et al., 2007, 2008; Slone et al., 2007), and "bitter" clades (Wanner and Robertson, 2008; Lee et al., 2009). To date, much attention has been paid to the gustatory receptors of Drosophila (Dahanukar et al., 2001, 2007; Dunipace et al., 2001; Slone et al., 2007; Jiao et al., 2007, 2008; Gardiner et al., 2008; Lee et al., 2010, 2012;; Weiss et al., 2011), but with rapid progress of genome projects on other insect species such as Anopheles gambiae (Hill et al., 2002), Bombyx mori (Wanner and Robertson, 2008), Tribolium castaneum (Richards et al., 2008), Apis mellifera (Robertson and Wanner, 2006), and Acyrthosiphon pisum (Smadja et al., 2009), the research is extending to a diverse range of species. However, rarely are studies on gustatory receptors carried out on serious agricultural pests such as Helicoverpa armigera.

The cotton bollworm, H. arimgera (Hübner), is one of the most destructive insect species. The larvae feed on numerous important cultivated crops such as cotton, peanuts, soybeans, 
and maize. Helicoverpa armigera is distributed widely in Asia, Africa, Europe, and Australia, and causes approximately US\$ 2 billion annual losses worldwide despite the use of insecticides (Sharm, 2001). The study of the H. armigera gustatory system may elucidate the underlying mechanisms that influence its feeding behavior and help to develop new insect-control strategies for such polyphagous pests.

In this study, we report the first gustatory receptor, HaGR9, from $H$. armigera. Further, we expressed HaGR9 in insect cells, and functionally characterized its topology and responses to substrates relevant to feeding behavior.

\section{Methods and Materials}

Insects and Cell Culture Helicoverpa armigera were fed artificial food in the laboratory under conditions described previously (Akhurst et al., 2003). Spodoptera frugiperda Sf9 cells (Invitrogen, USA) were cultured in Sf-900 II SFM medium according to the manufacturer's instructions. Drosophila melanogaster Schneider's S2 cells were maintained as a suspension culture in Drosophila Schneider's medium (Invitrogen, USA) adapting cells from $10 \%$ fetal bovine serum (FBS, Invitrogen, USA) to $1 \%$ FBS according to the Serum Halving Method. Cells were incubated at $28^{\circ} \mathrm{C}$ and subcultured to a final density of $1 \sim 2 \times 10^{6}$ cells/ $\mathrm{ml}$ when they reached a density of $\sim 6-10 \times 10^{6}$ cells $/ \mathrm{ml}$ (Mather and Roberts, 1998).

RNA Isolation, cDNA Synthesis and PCR Approximately fifty antennae, fifty mouthparts, ten foreguts, five midguts, and ten hindguts were dissected from mixed sex 5th instars. Approximately fifty tarsi and antennae were collected from male and female adults $(\mathrm{d} 1-\mathrm{d} 5)$. All collected tissues were stored immediately in RNA Later (Invitrogen, USA). Total RNA was purified using RNeasy (Qiagen, USA) or RNAqueous (Ambion, USA) kits according to the manufacturer's protocol. The purified RNA was treated with DNase I, quantified, and qualified by a NanoDrop ND-2000 (Thermo Scientific, USA), and a 2100 Bioanalyzer (Agilent, USA). The cDNA was synthesized using a SMART RACE (Rapid Amplification of cDNA End) cDNA amplification kit (BD Sciences, Clontech, USA) with SuperScript II reverse transcriptase (Invitrogen, USA), according to the manufacturer's manual. RT-PCR was performed for 40 cycles with an annealing temperature of $55{ }^{\circ} \mathrm{C}$ with primers (HaGR9-F CGCATGCTTTTATACTTAGG and HaGR9-R TCTCAATTGTCGTATCTTTGG). These primers spanned the known cDNA sequence and resulted in a $1300 \mathrm{bp}$ fragment. 5' RACE PCR was performed according to the SMART RACE cDNA amplification kit manual with the universal primer and gene-specific primers (HaGR9-1 GCTGACCGTGAAGCCATTGCTGCGAG and HaGR9-2
CTTGAGATCCTAAGTATAAAAGCATGCGCTCCGC). PCR products were purified using QIAquick gel extraction reagents (Qiagen, USA), cloned into the pGEM-T Easy vector (Promega, USA) and sequenced.

Immunocytochemistry Sf9 or S2 cells were subcultured on poly-L-Lysine-coated coverslips in 6-well plates and transfected with $1 \mu \mathrm{g}$ plasmid constructs and $6 \mu \mathrm{l}$ of Fugene HD transfection reagent (Promega, USA) in $200 \mu$ of medium per well. After $48 \mathrm{~h}$ post transfection, immunofluorescence under permeabilized and non-permeabilized conditions were performed as previously described (Zhang et al., 2011).

Calcium Imaging Sf9 cells were plated into 12-well plates and left to settle for $20 \mathrm{~min}$ before being transfected with $500 \mathrm{ng}$ of plasmid construct (PIB/V5-His vector as control, HaGR9 or MYC-epitope tagged HaGR9) and $3 \mu$ l of Fugene HD transfection reagent (Promega, USA) in $100 \mu$ l of medium per well. After $48 \mathrm{~h}$ post transfection, calcium imaging and data analysis were performed using a modification of a previously described method (Zhang et al., 2011; Anderson et al., 2009).

Tastants The tastants tested were D-fructose, D-galactose, D-glucose, sucrose, D-maltose, D-trehalose, and myo-inositol with purities $\geq 99 \%$. D-galactose was purchased from Amresco (USA), whereas the others were purchased from Sigma-Aldrich (USA). The maximum final concentration of tastants in each well was $50 \mathrm{mM}$. For dose-dependent calcium imaging studies, D-fructose, D-galactose, and Dmaltose were diluted with HBSS buffer (Zhang et al., 2011).

\section{Results}

Molecular Cloning and Expression Profile of HaGR9 Using DmGR43a amino acid sequence we searched in GenBank with Blastp (Altschul et al., 1990). A partial sequence of H. armigera olfactory receptor 4 (HaOR4) was found (EU818703) that contained 432 amino acids and lacked the N-terminal sequence. We studied its expression profile in several tissues: foregut, midgut, hindgut, antennae, and mouthparts from 5thinstars, as well as antennae and tarsi from male and female adults. PCR with gene-specific primers amplified a strong band of $1300 \mathrm{bp}$ from the larvae foregut (Fig. 1). A weak band also appeared in male antennae, larvae antennae, midgut, as well as the hindgut. Interestingly, there was no expression seen in the female antennae. However when the number of PCR cycles was increased from 40 to 50 (data not shown), we were able to detect a weak band, thus suggesting there is a low level of expression in the female antennae. The cDNA sample from foregut was used in 5' RACE reactions to clone the full-length sequence. 


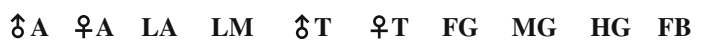

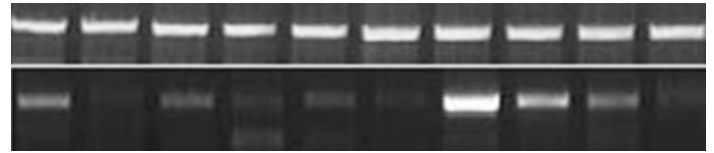

Actin

HaGR9

Fig. 1 RT-PCR analysis of HaGR9 gene expression in adult and larval tissues of Helicoverpa armigera. $\widehat{A} \mathrm{~A}$, Male adult antennae; $+\mathrm{A}$, Female adult antennae; LA, Larvae antennae, LM, Larvae mouthpart; $\mathrm{J}_{\mathrm{T}}^{\mathrm{T}}$, Male adult tarsi; O T, Female adult tarsi; FG, Foregut; MG, Midgut; HG, Hindgut; FB, Fat body. The actin gene was used as a control to qualify and quantify cDNA samples

The full-length sequence was cloned and named HaGR9 (JX970522) because of its $69 \%$ amino acid identity and $78 \%$ similarity to BmGR9. The predicted protein contains 465 amino acids with seven predicted transmembrane domains (Split 4.0 Server, http://split.pmfst.hr/split/4/) (Juretic et al., 2002). It also showed $26 \%$ amino acid identity and $49 \%$ similarity to DmGR43a. We performed a phylogenetic analysis on HaGR9 with 13 other insect GR43a-like receptors (Fig. 2). These insect GR43a-like receptors were divided into 4 order-specific groups: Diptera, Coleoptera, Hymenoptera, and Lepidoptera (Fig. 2). To date, five GR43a-like receptors have been identified from Lepidoptera insects, Papilio xuthus (Ozaki et al., 2011), Danaus plexippus (Zhan et al., 2011), B. mori (Wanner and Robertson, 2008; Sato et al., 2011;), Heliothis virescens (Krieger et al., 2002) and H. armigera. Due to sequence homology exclusively with DmGR43a rather than any insect olfactory receptors, the two receptor genes from $H$. virescens and $D$. plexippus reported previously most likely have been misclassified as odorant receptor 4 (Krieger et al., 2002; Zhan et al., 2011). The phylogenetic analysis (Fig. 2) showed that GR43a-like genes are widely distributed in various insect species, including almost all species with a sequenced genome. We also found a GR43a-like protein from pea aphid, A. pisum (XP_003244306), but its sequence is much longer (756 amino acids) than all the other known GR43a-like receptors ( $\sim 300-500$ amino acids), and, therefore, we did not include it in our phylogenetic analysis (Fig. 2).

Topology of HaGR9 The algorithm Split4.0 predicts seven transmembrane domains for HaGR9 with an intracellular Nterminus and an extracellular $\mathrm{C}$-terminus. To validate the topology we used immunofluorescence as previously described (Zhang et al., 2011). HaGR9 genes were fused to double MYC-epitope tags at either the N- or C-termini and expressed in both $\mathrm{S} 2$ cells and $\mathrm{Sf} 9$ cells. The native receptor (HaGR9) was used as a negative control. No positive signals were found from control cells (Figs.3b, c). Strong green fluorescence was visualized from cells transfected with either the N- or C-terminal labelled HaGR9 under permeabilized conditions (Figs. 3b, c). In contrast, green fluorescence could be detected only from cells transfected with C-terminally tagged HaGR9 (HaGR9:MYC) but not from cells transfected with N-terminally tagged HaGR9 (MYC:HaGR9) under unpermeabilized conditions. These results indicated that the $\mathrm{N}$-terminus of HaGR9 is intracellular and the C-terminus is

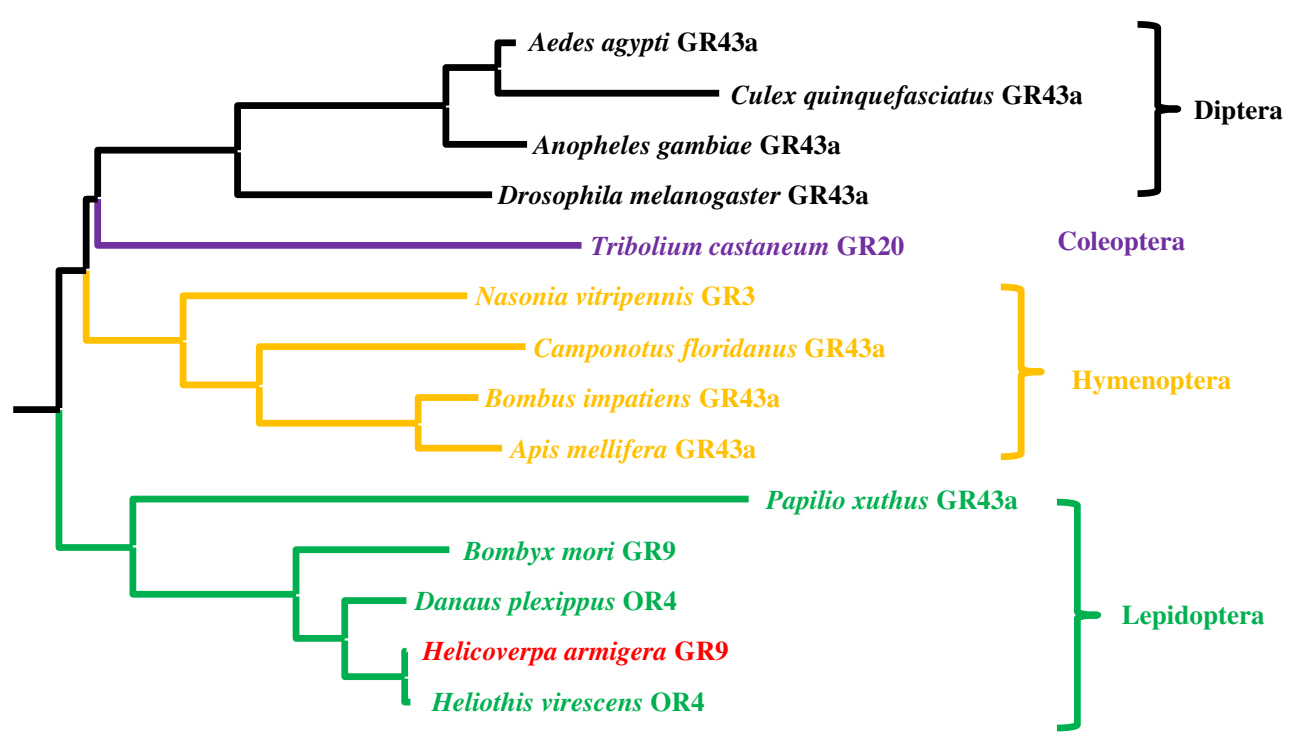

Fig. 2 Phylogenetic analysis of GR43a-like receptor genes from insects. The analysed genes included: Heliothis virescens OR4 (CAD31946) (Krieger et al., 2002), Danaus plexippus OR4 (EHJ77681) (Zhan et al., 2011), Bombyx mori GR9 (NP 001124345) (Sato et al., 2011) (Wanner and Robertson, 2008), Bombus impatiens GR43a (XP 003486787), Nasonia vitripennisGR3 (NP 001164386) (Robertson et al., 2010), Apis mellifera GR43a (XP_001121326),
Drosophila melanogaster GR43a (NP 523650) (Sato et al., 2011), Tribolium castaneum GR20 (EFA05758), Anopheles gambiae GR43a (XP_318100), Camponotus floridanus GR43a (EFN61344), Aedes aegypti GR43a (XP_001658898), Culex quinquefasciatus GR43a (XP_001842305), and Papilio xuthus GPCR (BAF91710) (Ozaki et al., 2011$)$ 
Fig. 3 HaGR9 has extracellular $\mathrm{C}$-terminus and intracellular $\mathrm{N}$-terminus indicated by immunofluorescence in both $\mathrm{Sf} 9$ and S2 cells. HaGR9 was expressed in its native form or fused with two MYC-epitope tags at either the $\mathrm{N}$ - or C-terminus. a Expression constructs for native HaGR9 (controls), N-terminally MYC-epitope tagged HaGR9 (MYC:HaGR9), and Cterminally MYC-epitope tagged HaGR9 (HaGR9:MYC). b, c Immunofluorescence of HaGR9 in Sf9 and S2 cells under permeabilized and unpermeabilized conditions. Green indicates the location of MYCepitope expression directed Alexa 488 fluorescence. Red indicates cell nuclear staining by DAPI. Scale bar $=5 \mu \mathrm{m}$ a

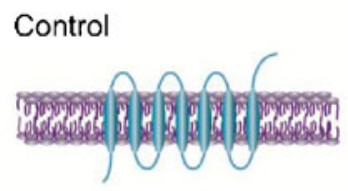

b

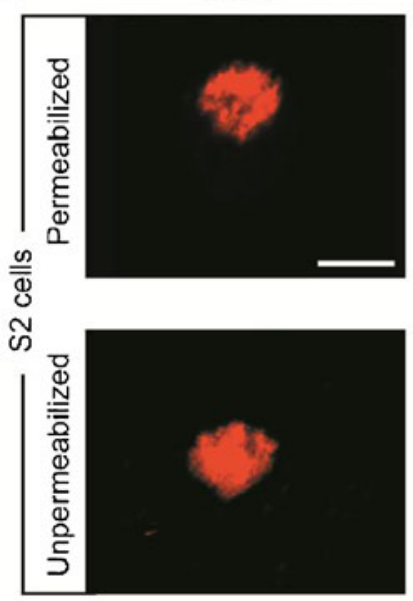

C

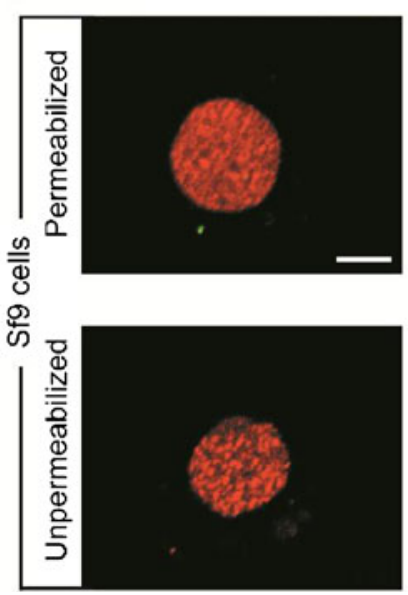

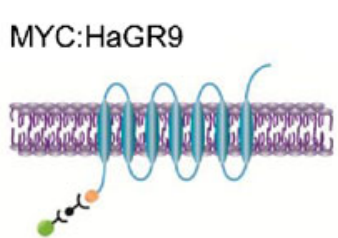

MYC:HaGR9
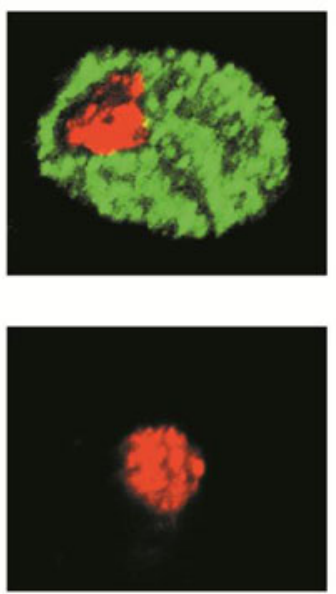

MYC:HaGR9
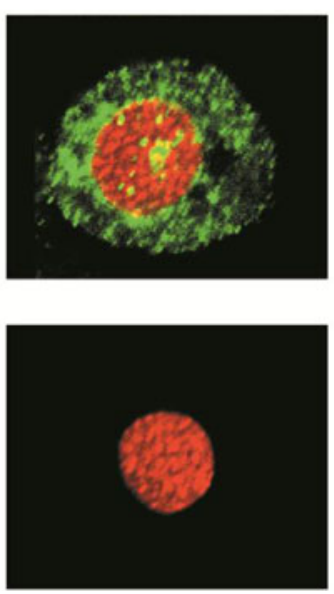

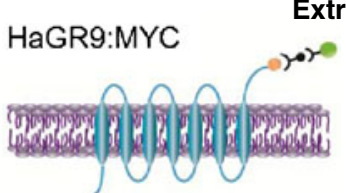

Extracellular

Cytoplasm
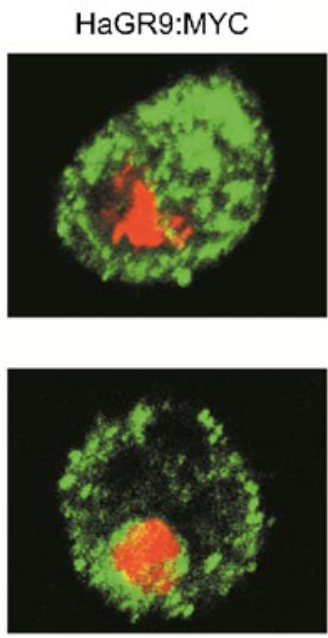

HaGR9:MYC
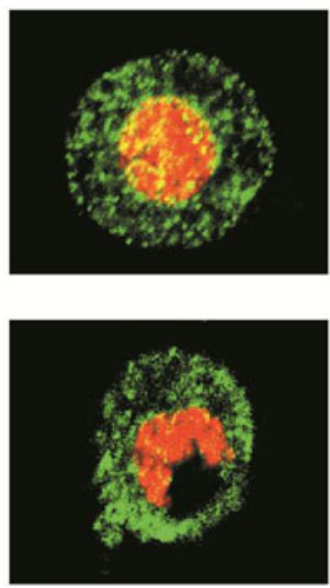

extracellular (Fig. 3a). The previous study of two BmGRs (BmGR8 and BmGR53 from the 'sugar' and 'bitter' clades) also showed that the N-terminus was inside the cell and the Cterminus located outside of the cell (Zhang et al., 2011), which is the same topology as observed for insect odorant receptors, but reverse to classical GPCRs (Benton et al., 2006). Insect receptor topologies previously were performed mainly on S2 cells from Drosophila (Smart et al., 2008; Zhang et al., 2011). Here, we used S2 cells (Fig. 3b) as well as Lepidoptera derived Sf9 cells (Fig. 3c). Both cell lines showed the same topology results, indicating both lines are suitable for insect receptor topology studies.

HaGR9 Can Detect Three Sugars We expressed HaGR9 in Sf9 cells and performed quantitative calcium imaging to characterize its specific ligand. Seven sugars, widespread in plant saps, and belonging to the monosaccharides (Dglucose, D-fructose, and D-galactose), disaccharides (sucrose, D-maltose, and D-trehalose), and myo-inositol were tested at $50 \mathrm{mM}$ in the initial screen. Three of them, Dgalactose, D-maltose, and D-fructose, generated responses that significantly differed from the control (Fig. 4a). A twotailed Student's $t$-test indicated that the order of responses of HaGR9 to three sugars is D-galactose $(\Delta \mathrm{F}=0.256, t=7.74$, $P<0.001)>$ D-maltose $(\Delta \mathrm{F}=0.211, t=10.78, P<0.001)>\mathrm{D}$ fructose $(\Delta \mathrm{F}=0.122, t=3.67, P<0.001)$. Our results also indicated that Sf9 cells themselves, although transfected with the empty expression vector, can be activated by the sugars, which we assume is due to endogenous receptors expressed in the Sf9 cell membrane. These responses vary 
a

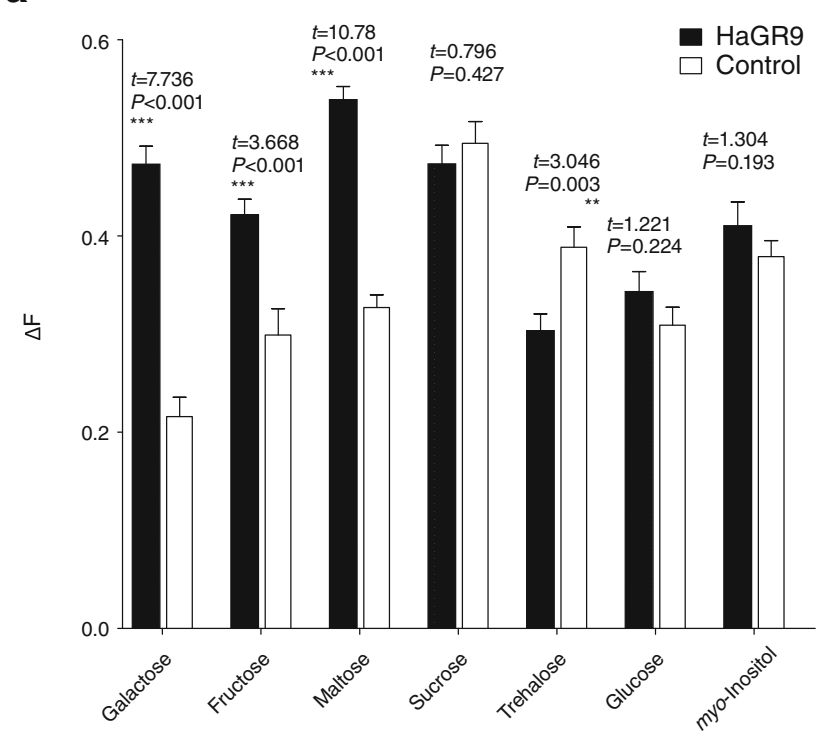

C

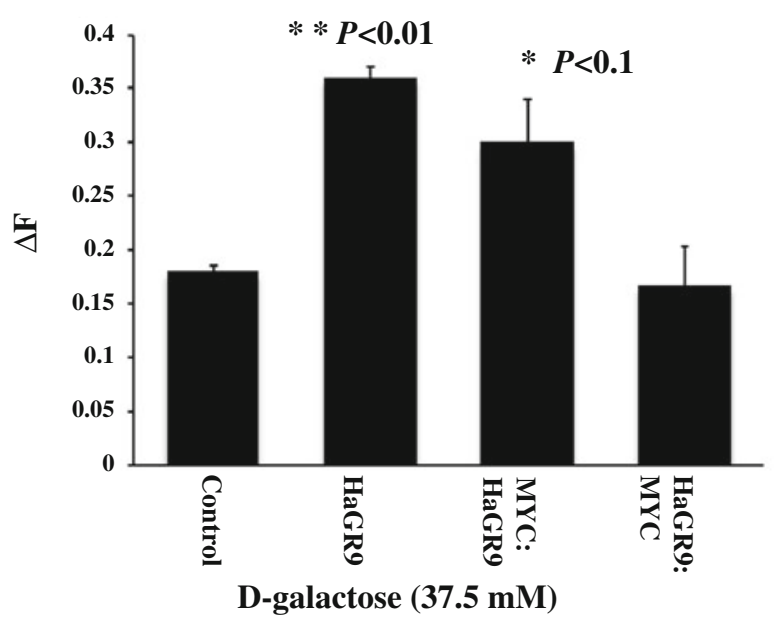

Fig. 4 Responsiveness of HaGR9 to sugars. a Responses of HaGR9 to seven tastants at $50 \mathrm{mM}$. The mean responses of cells expressing HaGR9 are indicated by the dark solid bar. The mean responses of controls are indicated by the black hollow bars. Error bars indicate the standard error of the mean. Analysis of the statistical significance between each response and control was conducted by two-tailed Student's $t$-test using arcsine transformation. $* * * P \leq 0.001, * * P<0.01$, $* P<0.05$. b Log dose-dependent response curves for HaGR9 to

based on the different sugars (Fig. 4a). Interestingly, cells transfected with HaGR9 showed reduced responses to trehalose when compared with responses from control Sf9 cells (Fig. 4a). We assume that the expression of HaGR9 may reduce or inhibit the expression, localization, or function of the endogenous taste receptors of Sf9 cells, thus leading to the lower responses to trehalose (Fig. 4a). Dosedependent responses to D-galactose, D-maltose, and Dfructose were performed (Fig. 4b). Results showed that these three sugars can cause similar dose-dependent responses from $5 \mathrm{mM}$ to $50 \mathrm{mM}$ concentrations (Fig. $4 \mathrm{~b}$ ) with similar $\mathrm{EC}_{50}$ b
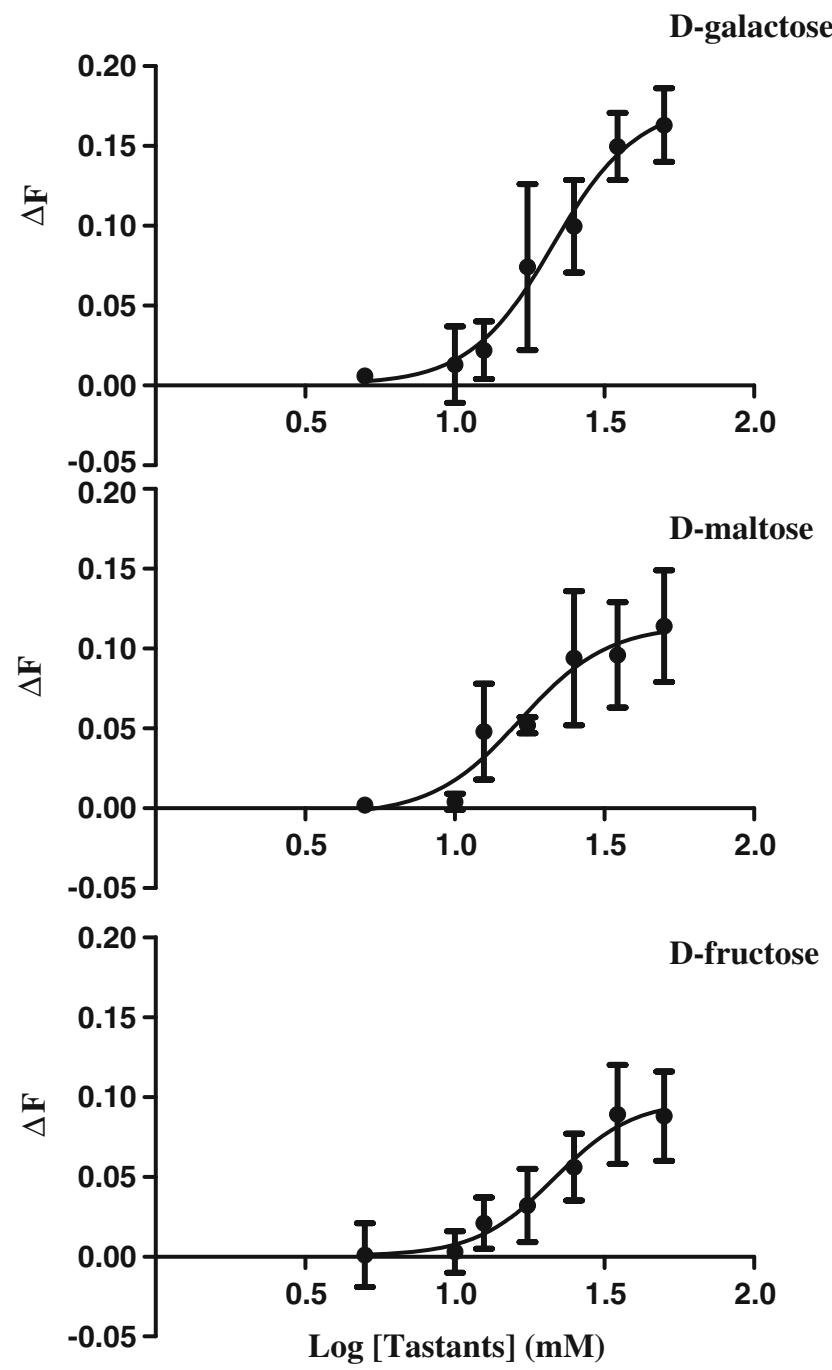

D-fructose, D-galactose, and D-maltose. The average responses of control cells have been subtracted. Error bars indicate the calculated error of the difference between means $=S E\left(\bar{x}_{G r 12}-\bar{x}_{\text {control }}\right)$. c Response of MYCepitope tagged HaGR9 to $37.5 \mathrm{mM}$ sugars. Control, empty vector (PIB/ V5-His vector); HaGR9, HaGR9-transfected cells; MYC:GR9, two MYC-epitope copies fused to HaGR9 N-terminus; GR9:MYC, two MYC-epitope copies fused to HaGR9 C-terminus

(21.3 $\pm 1.1 \mathrm{mM}$ for D-galactose, $16.5 \pm 1.3 \mathrm{mM}$ for D-maltose, and $21.5 \pm 1.1 \mathrm{mM}$ for D-fructose).

In order to determine whether the modified GRs used for topology studies retained functionality, identical calcium imaging experiments were performed (Fig. 4c). Cells transfected with native HaGR9 and N-terminally labelled HaGR9 (MYC:HaGR9) both showed significant responses when compared to empty vector controls (Fig. 3b). However, there were no statistically significant differences in responses of C-terminally labelled HaGR9 (HaGR9:MYC) and the negative control. We cannot rule out the possibility that 
the labelled HaGR9 renders the protein non-functional due to the incorrect insertion of the receptor into the membranes. A previous study on BmGR8 showed similar results where the N-terminally labelled tag did not significantly affect the receptor's function to detect myo-inositol (Zhang et al., 2011). However the C-terminally tagged receptor showed barely detectable function. These results indicate that the C-terminus of GRs is critical for their correct localisation and/or function.

\section{Discussion}

Our phylogenetic analysis (Fig. 2) demonstrates that GR43a is a conserved GR that exists in almost all insect species (20$64 \%$ amino acid identity), and therefore, GR43a may have a conserved function across insect orders. Our RT-PCR results (Fig. 1) indicate that HaGR9, a GR43a homologue, is highly transcribed in the larval foregut of $H$. armigera, where food is stored before moving into the midgut for digestion. A behavioral study on the blowfly, Phormia regina, showed the involvement of the foregut in the regulation of sugar taste threshold (Gelperin, 1966), with high sugar concentrations prolonging foregut stimulation and elevating the threshold for stimulation. Fructose and other sugars were shown to produce this behavioral effect when acting via the postulated foregut receptor (Gelperin, 1966). It is likely that HaGR9 may be a foregut receptor that is involved in the regulation of foregut stimulation and taste threshold in the digestive system, as well as in sugar sensing in external tissues. BmGR9, a GR43a ortholog from B. mori, also was shown to have high expression in the gut and respond to fructose significantly in vitro (Sato et al., 2011).

Recent studies in Drosophila show 14 gustatory receptors are expressed in the Drosophila gut, including GR43a, the fructose receptor, and GR64a from the sugar receptor family (Park and Kwon, 2011). The colocalization of the GR-Gal4 drivers with the regulatory peptides neuropeptide F (NPF), locustatachykinin (LTK), and diuretic hormone 31 (DH31) also was observed in this study (Park and Kwon, 2011), providing further evidence that gustatory receptors may be involved in the detection and regulation of nutrients during digestion. Gastrointestinal chemosensation is an emerging field in humans and other mammals, where gustatory receptors expressed in the luminal epithelium are believed to be acting as nutrient sensors to guide digestive processes, to protect from harmful substances, and to regulate food uptake (Sclafani, 2007; Breer et al., 2012). Therefore, an improved understanding of the intestinal chemosensation mechanisms may assist in the development of novel treatments of eating disorders, obesity, diabetes, intoxication, and inflammation (Breer et al., 2012). Insect digestive systems have many similarities to those of vertebrates in basic structure, cell types, and development, and may provide a simple system to study intestinal chemosensation (Park and Kwon, 2011).

To study the topologies of HaGR9, we used both Sf9 and S2 cell systems. Both gave identical results, showing HaGR9, a member of the DmGR43a-like subfamily, has an intracellular N-terminus and an extracellular C-terminus. This result is consistent with previous studies on other insect gustatory receptors from the sugar and bitter subfamilies (Zhang et al., 2011) and also with odorant receptors (ORs) (Benton et al., 2006; Lundin et al., 2007; Smart et al., 2008), which have been shown to be an evolutionarily related family (Robertson et al., 2003). Previous studies suggest that the GR gene family is an ancient chemoreceptor family from which a branch of OR genes subsequently evolved (Robertson et al., 2003). Insect ORs are thought to function as odor-gated ion channels (Sato et al., 2008), although a modulatory role for $G$ proteins and second messengers is likely to be involved in the downstream functions (Benton, 2008; Nakagawa and Vosshall, 2009). Here, we have shown that HaGR9 shares the same inverted topology as insect ORs, indicating that insect GRs are not classical GPCRs and may function in a way similar to insect ORs.

Genetic studies on Drosophila have revealed that coexpression of multiple GRs is essential for the detection of compounds like $\mathrm{CO}_{2}$, caffeine, theophylline, sucrose, Dglucose, and trehalose (Dahanukar et al., 2001,2007; Moon et al., 2006; Jones et al., 2007; Jiao et al., 2008 ). However, in vitro studies with BmGR8 (Zhang et al., 2011), BmGR9 and DmGR43a (Sato et al., 2011) have shown their responses to myo-inositol or D-fructose did not require the coexpression of other GRs, which is consistent with the HaGR9 studies reported here. We cannot conclude that HaGR9 can function without a co-receptor in vivo because $\mathrm{Sf} 9$ cells may express a native receptor that can assist HaGR9 in the detection of the three tested sugars. Orco, a highly-conserved OR co-receptor, was detected in $\mathrm{Sf} 9$ cells in a previous study (Smart et al., 2008), and is probably involved in the correct functioning of odorant receptors in the assay. In other in vitro studies using Xenopus oocytes, BmGR9 and DmGR43a showed a response only to D-fructose but not to other sugar tastants (Sato et al., 2011). HaGR9, the orthologous gene of BmGR9 and DmGR43a, showed responses to D-fructose, D-galactose, and D-maltose in our study. There may be two possibilities for these differences. First, HaGR9 shows $69 \%$ and $26 \%$ identity to BmGR9 and DmGR43a at the amino acid level, so it is possible the ligand binding capabilities differ. Second, Sf9 cells and Xenopus oocytes are different cell systems that may contain divergent components, leading to different activities and responses in the assay. To date, four major systems have been used to study insect chemosensory receptors: the "empty neuron" from Drosophila (Dobritsa et al., 2003), Xenopus oocytes with patch clamp technologies (Sakurai et al., 2004), human 
cells (HEK293T cells; Sato et al., 2008), and Lepidoptera cells ( $\mathrm{Sf9}$ cells) with calcium imaging (Smart et al., 2008; Anderson et al., 2009). We believe Sf9 cells are most suitable for studying Lepidoptera receptors as they are derived from a lepidopteran species, and therefore, are likely to more closely resemble in vivo $H$. armigera receptor function.

In summary, we here identified a novel insect-specific and insect-conserved gustatory receptor from H. armigera, and named it HaGR9. HaGR9 shows high homology to GR43a, and demonstrates high levels of transcription in the foregut of larvae. Topology study reveals this receptor has an intracellular N-terminus and an extracellular Cterminus, which is the same as previous studied insect GRs and ORs. In addition, HaGR9 shows dose-dependent responses to D-galactose, D-maltose, and D-fructose. Further immunohistochemistry studies to localize this receptor to specific cell types may shed light on the function of sugar receptors in the larval foregut. To study HaGR9 function in vivo, RNAi (Ozaki, et al., 2011) may be a powerful technique to inhibit its expression and perform feeding behavior assay. Studies on agricultural pests like $H$. armigera gustatory receptors may not only help us understand the underlying molecular mechanism of insect feeding behavior, but also help us develop new strategies to control this destructive species. For example, the studies on GR may direct the search for new attractants or repellents (Lee et al., 2010) from host plants that can be applied in pest control.

Acknowledgments We thank Joel Armstrong for providing $H$. armigera, Alexie Papanicolaou for bioinformatics help, Peter Campbell for RNA preparation. We also thank Alexie Papanicolaou, Bradley Stevenson, and Mira Dumancic for critically reading the manuscript. This work was supported by a CSIRO Julius Award (R-00094-01-005) and a CSIRO Office of the Chief Executive (OCE) post-doctoral fellowship.

Open AccessThis article is distributed under the terms of the Creative Commons Attribution License which permits any use, distribution, and reproduction in any medium, provided the original author(s) and the source are credited.

\section{References}

Akhurst, R. J., James, W., Bird, L. J., and Beard, C. 2003. Resistance to the Cry1Ac delta-endotoxin of Bacillus thuringiensis in the cotton bollworm, Helicoverpa armigera (Lepidoptera: Noctuidae). J. Econ. Entomol. 96:1290-1299.

Altschul, S. F., Gish, W., Miller, W., Myers, E. W., and LipMan, D. J. 1990. Basic local alignment search tool. J. Mol. Biol. 215:403-410.

Anderson, A. R., Wanner, K. W., Trowell, S. C., Warr, C. G., JaQuin-Joly, E., ZaGatti, P., Robertson, H., and NeWCOMB, R. D. 2009. Molecular basis of female-specific odorant responses in Bombyx mori. Insect. Biochem. Mol. Biol. 39:189-197.
Benton, R. 2008. Chemical sensing in Drosophila. Curr. Opin. Neurobiol. 18:357-363.

Benton, R., Sachse, S., Michnick, S. W., and Vosshall, L. B. 2006. Atypical membrane topology and heteromeric function of Drosophila odorant receptors in vivo. PLoS Biol 4:e20.

Breer, H., EBerle, J., FricK, C., HAID, D., and WidMAYER, P. 2012. Gastrointestinal chemosensation: chemosensory cells in the alimentary tract. Histochem. Cell Biol. 138:13-24.

ChyB, S., Dahanukar, A., Wickens, A., and Carlson, J. R. 2003. Drosophila Gr5a encodes a taste receptor tuned to trehalose. Proc. Natl. Acad. Sci. USA 100(Suppl 2):14526-14530.

Clyne, P. J., Warr, C. G., and CARLson, J. R. 2000. Candidate taste receptors in Drosophila. Science 287:1830-1834.

DahanuKar, A., Foster, K., van Der Goes VAn Naters, W. M., and CARLSON, J. R. 2001. A Gr receptor is required for response to the sugar trehalose in taste neurons of Drosophila. Nat. Neurosci. 4:1182-1186.

DahanUKar, A., LeI, Y. T., KwON, J. Y., and CARLSON, J. R. 2007. Two Gr genes underlie sugar reception in Drosophila. Neuron 56:503-516.

Dobritsa, A. A., van der Goes van Naters, W., Warr, C. G., StEINBRECHT, R. A., and CARLSON, J. R. 2003. Integrating the molecular and cellular basis of odor coding in the Drosophila antenna. Neuron 37:827-841.

Dunipace, L., Meister, S., McNealy, C., and Amrein, H. 2001. Spatially restricted expression of candidate taste receptors in the Drosophila gustatory system. Curr. Biol. 11:822-835.

GARDINER, A., BARKER, D., BUtLIN, R. K., JORDAN, W. C., and RitCHIE, M. G. 2008. Evolution of a complex locus: exon gain, loss and divergence at the Gr39a locus in Drosophila. PLoS One 3:e1513.

GELPERIN, A. 1966. Investigations of a foregut receptor essential to taste threshold regulation in the blowfly. J. Insect. Physiol. 12:829-841

Hill, C. A., Fox, A. N., Pitts, R. J., Kent, L. B., Tan, P. L., Chrystal, M. A., Cravchik, A., Collins, F. H., Robertson, H. M., and ZWIEBEL, L. J. 2002. G protein-coupled receptors in Anopheles gambiae. Science 298:176-178.

JiAO, Y., MoON, S. J., and Montell, C. 2007. A Drosophila gustatory receptor required for the responses to sucrose, glucose, and maltose identified by mRNA tagging. Proc. Natl. Acad. Sci. USA 104:14110-14115.

JiaO, Y., Moon, S. J., Wang, X., Ren, Q., and Montell, C. 2008. $\mathrm{Gr64f}$ is required in combination with other gustatory receptors for sugar detection in Drosophila. Curr. Biol. 18:1797-1801.

Jones, W. D., Cayirlioglu, P., Kadow, I. G., and Vosshall, L. B. 2007. Two chemosensory receptors together mediate carbon dioxide detection in Drosophila. Nature 445:86-90.

JuRETIC, D., ZorANIC, L., and ZUCIC, D. 2002. Basic charge clusters and predictions of membrane protein topology. J. Chem. Inf. Comput. Sci. 42:620-632.

Krieger, J., Raming, K., Dewer, Y. M., Bette, S., Conzelmann, S., and BREER, H. 2002. A divergent gene family encoding candidate olfactory receptors of the moth Heliothis virescens. Eur. J. Neurosci. 16:619-628.

Lee, Y., Kang, M. J., Shim, J., Cheong, C. U., Moon, S. J., and MONTELL, C. 2012. Gustatory receptors required for avoiding the insecticide L-canavanine. J. Neurosci. 32:1429-1435.

LEE, Y., KIM, S. H., and MonTELL, C. 2010. Avoiding DEET through insect gustatory receptors. Neuron 67:555-561.

Lee, Y., Moon, S. J., and Montell, C. 2009. Multiple gustatory receptors required for the caffeine response in Drosophila. Proc. Natl. Acad. Sci. USA 106:4495-4500.

Lundin, C., Kall, L., Kreher, S. A., Kapp, K., Sonnhammer, E. L., Carlson, J. R., Heijne, G., and Nilsson, I. 2007. Membrane topology of the Drosophila OR83b odorant receptor. FEBS Lett. 581:5601-5604. 
Mather, J. P. and Roberts, P. E. 1998. Introduction to Cell and Tissue Culture: Theory and Technique. Plenum Press, New York and London.

Moon, S. J., Kottgen, M., JiaO, Y., Xu, H., and Montell, C. 2006. A taste receptor required for the caffeine response in vivo. Curr. Biol. 16:1812-1817.

NaKagawa, T. and Vosshall, L. B. 2009. Controversy and consensus: noncanonical signaling mechanisms in the insect olfactory system. Curr. Opin. Neurobiol. 19:284-292.

OZAKI, K., RYUdA, M., YAMAdA, A., UtOGUChI, A., Ishimoto, H., Calas, D., Marion-Poll, F., Tanimura, T., and YoshikaWA, H. 2011. A gustatory receptor involved in host plant recognition for oviposition of a swallowtail butterfly. Nat. Commun. 2:542.

PARK, J. H. and KWON, J. Y. 2011. Heterogeneous expression of Drosophila gustatory receptors in enteroendocrine cells. PLoS One 6:e29022.

Richards, S., Gibbs, R. A., Weinstock, G. M., Brown, S. J., Denell, R., Beeman, R. W., Gibis, R., Bucher, G., Friedrich, M., GrimmelikhuiJzen, C. J., et al. 2008. The genome of the model beetle and pest Tribolium castaneum. Nature 452:949-955.

Robertson, H. M., Gadau, J., and Wanner, K. W. 2010. The insect chemoreceptor superfamily of the parasitoid jewel wasp Nasonia vitripennis. Insect. Mol. Biol. 19(Suppl 1):121-136.

ROBERTSON, H. M. and WANNER, K. W. 2006. The chemoreceptor superfamily in the honey bee, Apis mellifera: expansion of the odorant, but not gustatory, receptor family. Genome Res. 16:1395-1403.

Robertson, H. M., Warr, C. G., and Carlson, J. R. 2003. Molecular evolution of the insect chemoreceptor gene superfamily in Drosophila melanogaster. Proc. Natl. Acad. Sci. USA 100 (Suppl 2):14537-14542.

Sakurai, T., NaKagawa, T., Mitsuno, H., Mori, H., Endo, Y., Tanoue, S., Yasukochi, Y., Touhara, K., and Nishioka, T. 2004. Identification and functional characterization of a sex pheromone receptor in the silkmoth Bombyx mori. Proc. Natl. Acad. Sci. USA 101:16653-16658.
Sato, K., Pellegrino, M., Nakagawa, T., Vosshall, L. B., and TOUHARA, K. 2008. Insect olfactory receptors are heteromeric ligand-gated ion channels. Nature 452:1002-1006.

SATO, K., TANAKA, K., and TOUHARA, K. 2011. Sugar-regulated cation channel formed by an insect gustatory receptor. Proc. Natl. Acad. Sci. USA 108:11680-11685.

SClafani, A. 2007. Sweet taste signaling in the gut. Proc. Natl. Acad. Sci. USA 104:14887-14888.

Sharm, H. C. 2001. Cotton Bollworm/Legume Pod Borer, Helicoverpa armigera (Hubner) (Noctuidae: Lepidoptera): Biology and Management. Crop Protection Compendium. p. 77, Commonwealth Agricultural Bureau International Oxon, UK.

Slone, J., DANiels, J., and AMreIn, H. 2007. Sugar receptors in Drosophila. Curr. Biol. 17:1809-1816.

Smadja, C., Shi, P., Butlin, R. K., and Robertson, H. M. 2009. Large gene family expansions and adaptive evolution for odorant and gustatory receptors in the pea aphid, Acyrthosiphon pisum. Mol. Biol. Evol. 26:2073-2086.

Smart, R., Kiely, A., Beale, M., Vargas, E., Carraher, C., KraliceK, A. V., Christie, D. L., Chen, C., Newcomb, R. D., and WARR, C. G. 2008. Drosophila odorant receptors are novel seven transmembrane domain proteins that can signal independently of heterotrimeric G proteins. Insect. Biochem. Mol. Biol. 38:770-780.

WANNER, K. W. and ROBERTSON, H. M. 2008. The gustatory receptor family in the silkworm moth Bombyx mori is characterized by a large expansion of a single lineage of putative bitter receptors. Insect. Mol. Biol. 17:621-629.

Weiss, L. A., Dahanukar, A., Kwon, J. Y., BanerJee, D., and CARLSON, J. R. 2011. The molecular and cellular basis of bitter taste in Drosophila. Neuron 69:258-72.

Zhan, S., Merlin, C., Boore, J. L., and Reppert, S. M. 2011. The monarch butterfly genome yields insights into long-distance migration. Cell 147:1171-1185.

Zhang, H. J., Anderson, A. R., Trowell, S. C., Luo, A. R., Xiang, Z. H., and XIA, Q. Y. 2011. Topological and functional characterization of an insect gustatory receptor. PLoS One 6:e24111. 